\title{
Dynamic monitoring with shear wave elastography predicts outcomes of chronic hepatitis B patients with decompensated cirrhosis
}

\author{
Junzhao Ye ${ }^{1} \wedge$, Yang Huang ${ }^{2}$, Yanhong Sun ${ }^{3}$, Congxiang Shao ${ }^{1}$, Shenghong Zhang ${ }^{1}$, Wei Wang ${ }^{2}$, Bihui Zhong ${ }^{1 \wedge}$ \\ ${ }^{1}$ Department of Gastroenterology, The First Affiliated Hospital, Sun Yat-sen University, Guangzhou, China; ${ }^{2}$ Department of Ultrasound, The First \\ Affiliated Hospital, Sun Yat-sen University, Guangzhou, China; ${ }^{3}$ Department of Clinical Laboratory, The First Affiliated Hospital, Sun Yat-sen \\ University, Guangzhou, China \\ Contributions: (I) Conception and design: J Ye, B Zhong, W Wang; (II) Administrative support: B Zhong, W Wang, S Zhang; (III) Provision of study \\ materials or patients: Y Huang; (IV) Collection and assembly of data: W Wang, Y Sun, C Shao; (V) Data analysis and interpretation: J Ye, B Zhong; (VI) \\ Manuscript writing: All authors; (VII) Final approval of manuscript: All authors. \\ Correspondence to: Wei Wang, MD. Division of Interventional Ultrasound, Department of Medical Ultrasonics, The First Affiliated Hospital of Sun \\ Yat-sen University, No. 58 Zhongshan Road II, Yuexiu District, Guangzhou 510080, China. Email: wangw73@mail.sysu.edu.cn; Bihui Zhong, MD. \\ Department of Gastroenterology, The First Affiliated Hospital, Sun Yat-sen University, No. 58 Zhongshan Road II, Yuexiu District, Guangzhou \\ 510080, China. Email: zhongbh@mail.sysu.edu.cn.
}

Background: Two-dimensional shear wave elastography (2D-SWE) can be used to accurately assess significant liver fibrosis in chronic hepatitis B (CHB). However, whether those with decompensated cirrhosis could benefit from surveillance with 2D-SWE remains unclear. We aimed to evaluate the association between dynamic changes in 2D-SWE measurements and the prognosis of CHB-related decompensated cirrhosis.

Methods: We prospectively enrolled 149 consecutive treatment-naive CHB patients with decompensated cirrhosis from a clinical trial (registration number: ChiCTR-DCD-15006000) from February 2015 to December 2018. 2D-SWE was performed at 48-week intervals until December 2020. Liver-related events (LREs) were recorded through electronic medical records and telephone interviews.

Results: The liver stiffness measurement (LSM) levels persistently reduced after antiviral therapy in patients who did not develop LREs, while patients with LREs showed a fluctuating trend of LSM alterations. Low pre-treatment 2D-SWE LSM, $\Delta \%$ 2D-SWE LSM pre-virus control, and $\Delta \%$ 2D-SWE LSM prepost treatment were characterized by similar prognostic abilities as high levels of these parameters. Posttreatment 2D-SWE LSM was independently correlated with LREs in multivariate Cox regression models after antiviral treatments [hazard ratio $(\mathrm{HR})=1.05$; 95\% confidence interval $(\mathrm{CI}): 1.02-1.08, \mathrm{P}=0.0007$ and 1.11; 95\% CI: 1.04-1.18, P=0.0026]. Receiver operating characteristic (ROC) curve analysis identified that post-treatment 2D-SWE LSM exhibited the best predictive power for LREs among all the other variables, including parameters of 2D-SWE and serum fibrosis markers (area under the curve $=0.75, \mathrm{P}<0.001$ ).

Conclusions: The last follow-up LSM, rather than pre-treatment or dynamic changes in 2D-SWE serves as a powerful predictor of LREs, suggesting that sequential monitoring may be beneficial to predicting prognosis.

Keywords: Two-dimensional shear wave elastography (2D-SWE); liver stiffness measurements (LSMs); chronic hepatitis B (CHB); decompensated cirrhosis; prognosis

Submitted Jun 06, 2021. Accepted for publication Aug 31, 2021.

doi: $10.21037 / \mathrm{atm}-21-3142$

View this article at: https://dx.doi.org/10.21037/atm-21-3142

^ ORCID: Junzhao Ye, 0000-0003-3163-4164; Bihui Zhong, 0000-0002-4638-7699. 


\section{Introduction}

Chronic hepatitis B (CHB) virus infection is a major, global public health concern as over 257 million individuals are infected with $\mathrm{CHB}$, with 887,000 deaths annually (1). The development of decompensated cirrhosis from liver inflammation and fibrosis are major factors of liverrelated death during $\mathrm{CHB}$, predisposing patients with this infection to life-threatening complications including liver failure, portal vein hypertension related bleeding, spontaneous peritonitis, and primary hepatocellular cancer (HCC). Once CHB progresses to decompensated cirrhosis, the median survival time decreases from over 10 years in compensated cirrhosis to nearly 2 years (2). Effective antiviral treatment with nucleos(t)ide analogs (NUCs) can improve the prognosis of $\mathrm{CHB}$; however, the progression under virus control by NUCs is heterogeneous. Therefore, distinguishing patients who should receive more intensive treatments and monitoring is critical to the management of CHB with decompensated cirrhosis.

Liver stiffness measurements (LSM) with ultrasound elastography, which estimates the severity of fibrosis utilizing ultrasound imaging-based methods to detect the velocity of shear waves, is a simple noninvasive tool for predicting CHB-related decompensated cirrhosis complications in the Baveno VI Consensus (3). Baseline and regular LSM using transient elastography has been proven to be useful in the prediction of decompensated events such as portal hypertension progression for patients with compensated cirrhosis caused by hepatitis B virus (HBV) and some other etiologies (4). Furthermore, LSM in patients with $\mathrm{HBV}$-related compensated cirrhosis would decrease significantly after antiviral therapy, and these treatment-related changes in LSM could predict 2-year liver decompensation-related endpoints (5). However, most of the published studies reported the results in patients with CHB-related compensated cirrhosis. The value of LSM in populations with CHB-related decompensated cirrhosis monitoring remains unknown.

As a more recent elastography technique than transient elastography, two-dimensional shear wave elastography (2D-SWE) enables real-time visualization of the elastogram, a color rendering of liver stiffness, which can serve as an indicator of fibrosis severity over a larger detection area (up to $700 \mathrm{~mm}^{2}$ ) and minimizes the interruptions from ascites or obesity (6). A meta-analysis covering 13 studies and 1,143 patients demonstrated that 2D-SWE has a better performance for the noninvasive staging of liver fibrosis in patients with $\mathrm{CHB}$, with the area under the receiver operating characteristic (ROC) curve (AUC) increasing by $0.003-0.034$ when diagnosing cirrhosis $(\mathrm{P}=0.022)(7)$. However, in a recent report of a 4-year follow-up with rare liver-related events (LREs) (6.7\%), baseline LSM of 2D-SWE showed an AUC similar to that of transient elastography (AUC, 0.86 vs. $0.88, \mathrm{P}=0.71$ ) for the prediction of liver-related outcomes in patients with $\mathrm{CHB}$, which may ignore the value of dynamic changes of 2D-SWE (8). Therefore, it would be necessary to explore the predictive value of baseline and dynamic changes in 2D-SWE in hepatic complications for those with decompensated cirrhosis receiving antiviral therapy.

In the current study, we endeavored to validate the potential practical values of pre- and post-treatment 2D-SWE LSM levels and their dynamic changes for predicting liver endpoints in a prospective cohort of patients with $\mathrm{HBV}$-associated decompensated cirrhosis undergoing NUC treatments to shed light on the predictive power of this tool for prognosis assessment. We present the following article in accordance with the STARD reporting checklist (available at https://dx.doi.org/10.21037/atm-21-3142).

\section{Methods}

\section{Patients and study design}

This was a prospective observational study enrolling consecutive $\mathrm{CHB}$ patients from a clinical trial (registered in the Chinese Clinical Trial Register: ChiCTRDCD-15006000) (9) in the First Affiliated Hospital, Sun Yat-sen University between February 2015 and December 2018. The study was conducted in accordance with the Declaration of Helsinki (as revised in 2013). The study protocol was approved by the institutional ethics committee of The First Affiliated Hospital, Sun Yat-sen university $\{[2014] 112\}$, and all patients provided signed informed consent. The inclusion criteria were as follows: (I) persistent serum HBV DNA positivity for over 6 months; (II) clinical diagnosis of decompensated cirrhosis; and (III) antiviral treatment-naive patients. The exclusion criteria included: (I) coinfection with another hepatitis virus, (II) autoimmune liver disease, (III) alcoholic liver disease, and (IV) the presence of severe extrahepatic diseases or pregnancy. Clinically decompensated cirrhosis was diagnosed by radiologic evidence (computed tomography or magnetic resonance imaging found changes in liver morphology, such as nodules in the hepatic parenchyma and serrated changes 
on the liver surface) plus at least one feature of ascites, liver failure, variceal bleeding, hepatopulmonary syndrome, hepatorenal syndrome, or encephalopathy (10).

\section{D-SWE measurements}

2D-SWE was carried out using a Supersonic Imagine system (Aix-en-Provence, France) by two fixed sonographers with over 3 years of experience with 2D-SWE who were unaware of the aim of the study and blinded to other clinical information. In 50 cases the liver of patients with decompensated cirrhosis with $\mathrm{CHB}$ was measured by the same sonographer twice and by a second sonographer twice to calculate intra- and interobserver agreement before the study. In this study, they performed 2D-SWE measurements independently. The detailed procedure of performing 2D-SWE has been described in our previous study (9) and is briefly described as follows: 2D-SWE was performed in dual mode using a right intercostal approach for image collection. Under the guidance of conventional, real-time B-mode imaging, a rectangular electronic region of interest (ROI, approximately $4 \mathrm{~cm} \times 3 \mathrm{~cm}$ and set $1-2 \mathrm{~cm}$ under the liver surface) avoiding any focal lesion, vessels, biliary tracts, or artifacts was displayed on the best static SWE image and a circular ROI (the diameter $=2 \mathrm{~cm}$ ) inside was set in the center of the 2D-SWE elasticity box possibly for analysis (Figure S1). The operator aimed to achieve homogeneous color filling of the SWE ROI placed on the most homogeneous, stable elastogram area. Before this study, a preliminary study of $50 \mathrm{CHB}$ patients with decompensated cirrhosis in our center showed that the kappa statistic of the interobserver between the two sonographers and scan-rescan reproducibility for the same sonographer were 0.92 and 0.94 , respectively. Five consecutive 2D-SWE images were acquired, and the mean values were used for analysis.

\section{Clinical and laboratory parameters}

Demographics, drinking habits, HBV infection history, and medication information were obtained using a faceto-face interview and were recorded with a predetermined structured questionnaire [supplementary material of questionnaire (Appendix 1)]. Serum biochemical indexes were tested for the following measurements: alanine aminotransferase (ALT), aspartate transaminase (AST), glutamyl transferase (GGT), alkaline phosphatase, total bilirubin, direct bilirubin, albumin, alpha-fetoprotein (AFP), creatinine, and prothrombin time, platelet. HBV DNA levels were assessed by Roche $\mathrm{COBAS}^{\circledR} \mathrm{TaqMan}^{\circledR} \mathrm{HBV}$ Test. Hepatitis B surface antigen (HBsAg) and hepatitis B envelope antigen ( $\mathrm{HBeAg}$ ) were quantified by Elecsys (Roche Diagnostics GmbH, Mannheim, Germany). The model end-stage liver disease (MELD) score was calculated using the following formula: $3.8 \times \ln$ [bilirubin $(\mathrm{mg} / \mathrm{dL})$ ] $+11.2 \times \ln [$ international normalized ratio (INR)] $+9.6 \times$ $\ln [$ creatinine $(\mathrm{mg} / \mathrm{dL})]+6.4$ (11). Child-Pugh score was classified based on the grading of the bilirubin, albumin, ascites, encephalopathy, and INR, according to the literature (12).

\section{Serum fibrosis markers measurements}

Concentrations of serum fibrosis markers including hyaluronic acid (HA), procollagen III amino terminal propeptide (PIIINP), laminin (LN) and collagen type IV (CIV) were measured with magnetic particle chemiluminescence enzyme-linked immunoassay (Autobio Diagnostic Co, Zhengzhou, China). Fibronectin (FN) and haptoglobin (HP) were measured with immunoturbidimetry (Szybio, Shanghai, China).

\section{Follow-up and study endpoints}

All patients initiated antiviral treatment at baseline with entecavir or tenofovir disoproxil fumarate and maintained it until the last follow-up (December, 2020). Some patients received entecavir and the others chose tenofovir as the latter was available in China until 2017. During antiviral treatment, conventional medical therapy was offered in accordance with standard clinical practice $(13,14)$. The follow-up was counted from the baseline. Follow-up for blood cell count, liver biochemistry test, HBV viral markers, AFP, and prothrombin activity (PTA), was conducted every 3 months, while 2D-SWE and serum fibrosis marker panels were measured every $48 \pm 4$ weeks. The relative change parameters of $2 \mathrm{D}$-SWE monitoring $(\Delta \%)$ were defined as follows: (I) $\Delta \%$ 2D-SWE LSM pre-viral control =

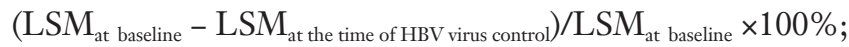
LSM at the time of HBV virus control represented the next follow-up measures of LSM after the HBV DNA decrease to lower than $20 \mathrm{IU} / \mathrm{m}$. (II) $\Delta \% 2 \mathrm{D}$-SWE LSM viral control-post $=\left(\mathrm{LSM}_{\text {at the time of viral control }}-\mathrm{LSM}_{\text {at the last follow-up }}\right) /$ $\mathrm{LSM}_{\text {at the time of viral control }} \times 100 \%$; (III) $\Delta \%$ previous yearly changes of $2 \mathrm{D}-\mathrm{SWE} \mathrm{LSM}=\left(\mathrm{LSM}_{\text {at the last second follow-up }}\right.$ $\left.\mathrm{LSM}_{\text {at the last follow-up }}\right) / \mathrm{LSM}_{\text {at the last second follow-up }} \times 100 \%$; (IV) $\Delta \%$ 
2D-SWE LSM pre-post $=\left(\mathrm{LSM}_{\text {at baseline }}-\mathrm{LSM}_{\text {at the last follow-up }}\right) /$ $\mathrm{LSM}_{\text {at baseline }} \times 100 \%$. The post-treated 2D-SWE LSM referred to the last follow-up LSM measurements during the yearly regular LSM follow-up after initiating antiviral therapy. The choice of the 4 dynamic indicators was based on the CHB related inflammation and stiffness could be improved by the antiviral treatment and therefore the response of LSMs of 2D-SWE at the time of viral control after antiviral treatment would be considered. Two authors (J Ye and C Shao) who were blinded to the aim of the study confirmed the date of disease progression by electronic hospital records or through telephone interview with the patient or their relatives until December 2020.

The primary study endpoint was the occurrence of live-related disease progression, including hepatic decompensation, hepatocellular carcinoma, or liver-related death $(15,16)$. Hepatic decompensation was defined as gastric or esophageal varices bleeding, spontaneous bacterial peritonitis, hepatic encephalopathy, hepatopulmonary syndrome, hepatorenal syndrome and liver failure, with established diagnosis according to European Association for the Study of the Liver (EASL) guidelines (15). For patients with multiple liver progression events, only the first event and the corresponding occurrence time were used for the analysis. HCC development was confirmed by ultrasoundguided tumor biopsy or postoperative pathology according to the Edmondson-Steiner criteria (17).

\section{Statistics}

Statistical analyses were processed using $\mathrm{R}$ language version 3.3.3. Variables are presented as the means \pm standard deviations or percentages. Quantitative data were compared using an independent-samples $t$-test or the Mann-Whitney test, while categorical data were compared using the Pearson chi-squared test. The two-way repeated measures ANOVA to investigated the changing trends differences between patients with and without LREs. The cumulative probabilities of developing of LREs were calculated using the Kaplan-Meier method. Univariable analyses using the log-rank test were computed to identify prognostic factors predictive of each event. A multivariate model was constructed with Cox regression to analyze the prognostic factors that had $\mathrm{P}<0.05$ or $2 \mathrm{D}-\mathrm{SWE}$-related parameters in univariate analysis and collinearity diagnostics (variance inflation factor <1.8). Significant cutoff values for factors of interest and their corresponding sensitivities, specificities and accuracies were determined using the ROC curve.
The optimal threshold of LSMs predicting LREs was determined to obtain a maximum sum of sensitivity and specificity. All analyses were two-sided, and $\mathrm{P}$ values $<0.05$ were considered significant.

\section{Results}

\section{Study cohort characteristics at baseline and during follow-up}

A total of 153 patients were enrolled, and four patients declined to participate. Consequently, 149 patients with $\mathrm{HBV}$-associated decompensated cirrhosis (mean age of $51.1 \pm 11.8$ years and $78.5 \%$ male) were included in this analysis, with a median follow-up of 34.8 months (Figure S2). The detailed follow-up flow chart is presented in Figure 1. The four patients lost to follow-up were ascribed to the LRE group for statistical analysis to lessen the overestimation of the changing trend of SWE in those without LRE and allow us to utilize their follow-up information. The detailed baseline characteristics are presented in Table 1 with the overall cohort grouped by LREs developed after follow-up. The patients with LREs were older than those without $(54.0 \pm 11.1$ vs. $49.6 \pm 11.9$ years, $\mathrm{P}=0.028)$, whereas no significant differences were found in the proportions of males, body mass index, levels of HBV viral markers, liver enzymes, liver functions (albumin, globulin, total bilirubin, and PTA), platelets or creatinine. Moreover, higher concentrations of LN $(112.7 \pm 103.9$ vs. $73.8 \pm 49.2 \mathrm{ng} / \mathrm{mL}$, $\mathrm{P}=0.020)$ and LSMs by $2 \mathrm{D}-\mathrm{SWE}(20.2 \pm 11.2 v s .17 .0 \pm 7.6 \mathrm{kPa}$, $\mathrm{P}=0.044)$ at baseline were shown in patients with LREs.

During the follow-up, no patients experienced 2D-SWE measurement failures (no color-coded elasticity images after five trials). During the follow-up period, 53 of the 149 patients (35.6\%) developed LREs. The most common event was spontaneous bacterial peritonitis in 38 patients, followed by variceal bleeding ( 24 patients), liver failure (16 patients, and all the hepatorenal syndrome (4 patients, $25 \%$ ), hepatopulmonary syndrome (2 patients, $12.5 \%$ ) occurred in these patients], hepatocellular carcinoma occurrence (4 patients), and 29 patients of them had at least two aforementioned complications. Eleven patients died of these complications.

For the status of patients at the last measurement of 2D-SWE before LRE occurrence or the follow-up finish (Table S1), those with LREs that developed after followup presented higher levels of GGT, PIIINP, LN, CIV and LSM of 2D-SWE than those without LREs, while no significant differences were found in the levels of HBV 


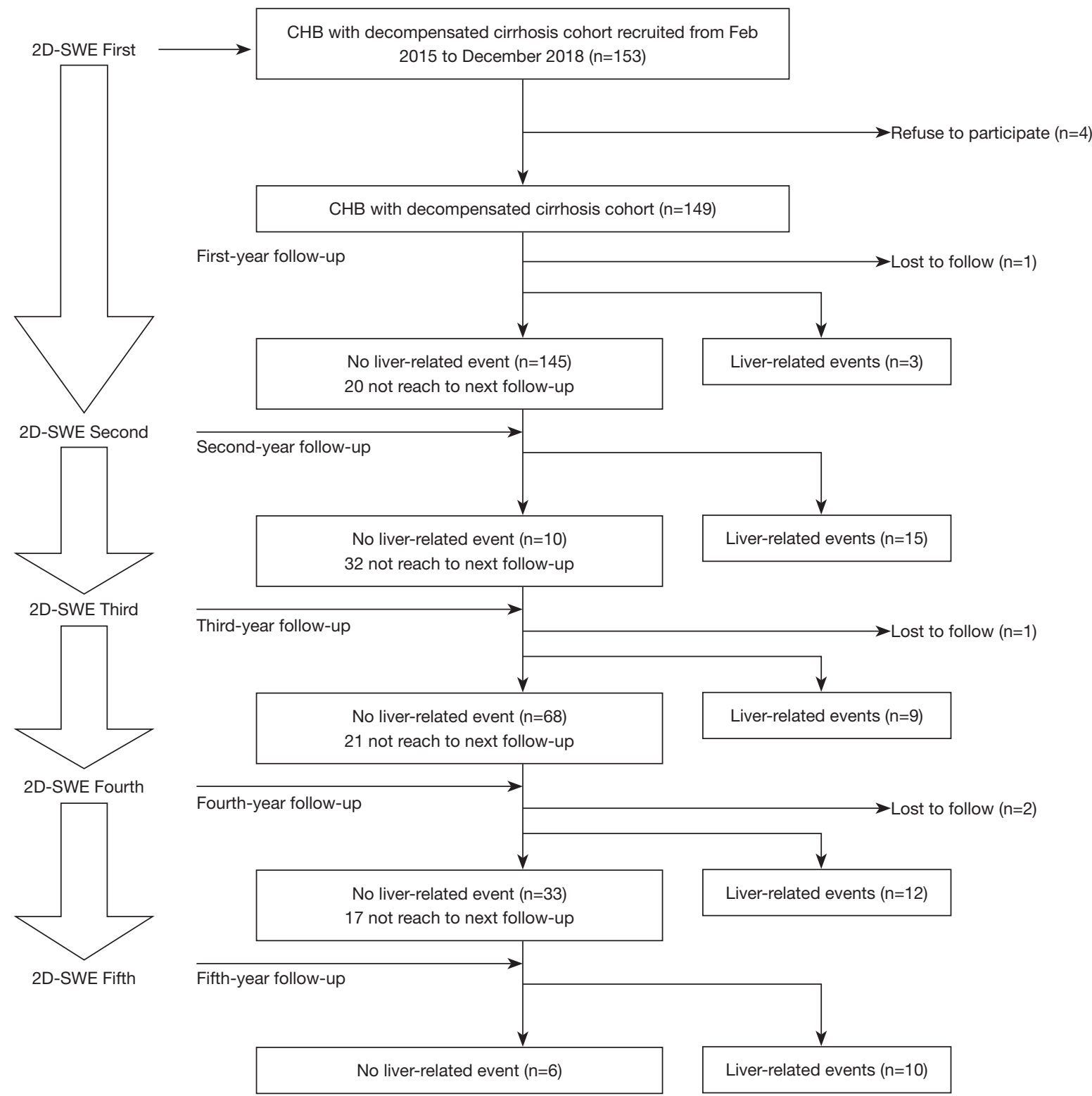

Figure 1 Flow chart of regular 2D-SWE monitoring during the study period for patients with CHB related decompensated cirrhosis undergoing antiviral therapy. One hundred ad forty-nine patients underwent initial 2D-SWE measurements and consequently there were 145, 110, 68, and 33 individuals received 2, 3, 4, and 5 repeated 2D-SWE measurements. Abbreviations: 2D-SWE, two-dimensional shear wave elastography; $\mathrm{CHB}$, chronic hepatitis $\mathrm{B}$.

related-markers, the other liver biochemical indexes or platelets between the two groups (Table S1).

\section{Changing patterns of regular 2D-SWE LSM monitoring between those with and without LREs}

For all patients, there were 1-5 (median: 3 ) measurements for LSMs by 2D-SWE in the study period. Those who had only one 2D-SWE measure would not be excluded, because their data would be utilized to analyze the associations between the baseline LSM and the outcomes. We analyzed the dynamic changes in LSMs by 2D-SWE in patients undergoing antiviral therapy (Figure $2 A, 2 B$ ). For patients without LREs, the mean LSM values 
Table 1 Baseline characteristics of patients with HBV-related decompensated cirrhosis stratified by liver-related events developed after follow-up

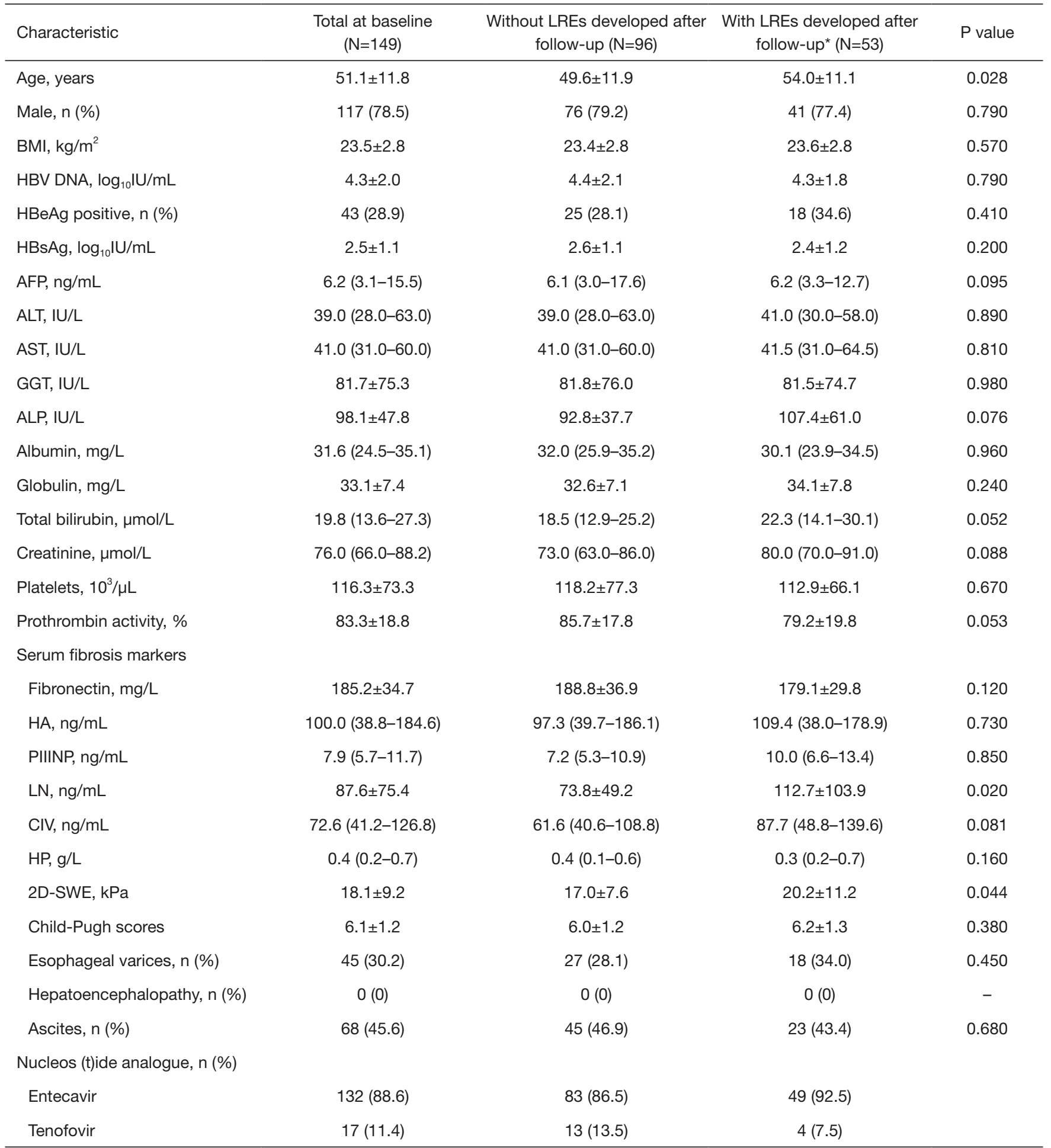

Normally and non-normally distributed variables were expressed as mean \pm standard deviation or median (25-75\% quantiles), respectively. ${ }^{*}$ the 4 patients lost to follow-up were ascribed to the LRE group for statistical analysis. Abbreviations: 2D-SWE, two-dimensional shear wave elastography; AFP, alpha-fetoprotein; ALP, alkaline phosphatase; ALT, alanine aminotransferase; AST, aspartate transaminase; BMI, body mass index; CIV, collagen type IV; GGT, glutamyl transferase; HA, hyaluronic acid; HBeAg, hepatitis B envelope antigen; HBsAg, hepatitis B surface antigen; HBV, hepatitis B virus; HP, haptoglobin; LN, laminin; LRE, liver-related event; PIIINP, procollagen III amino terminal propeptide. 
A
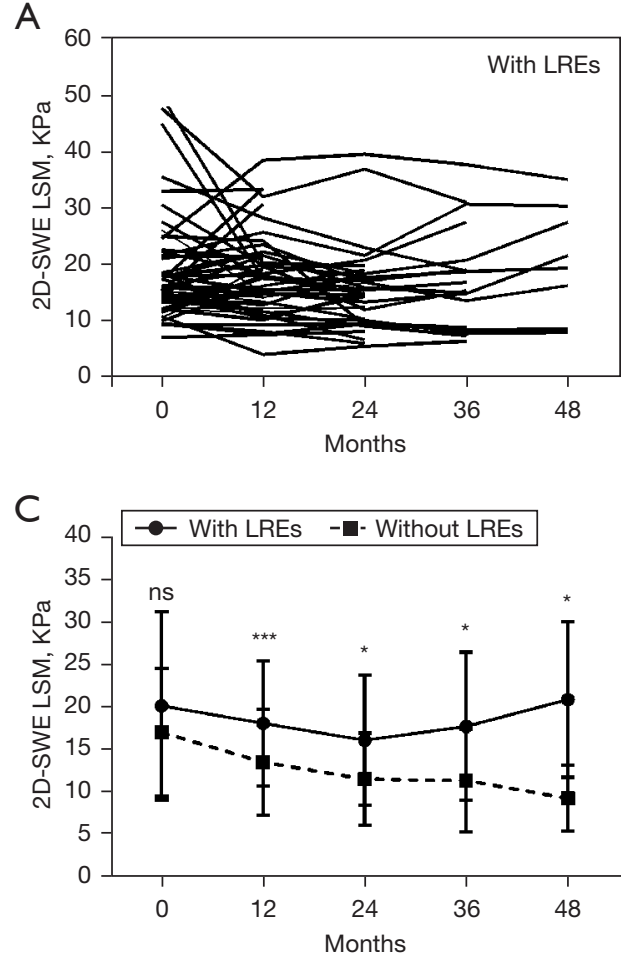

B
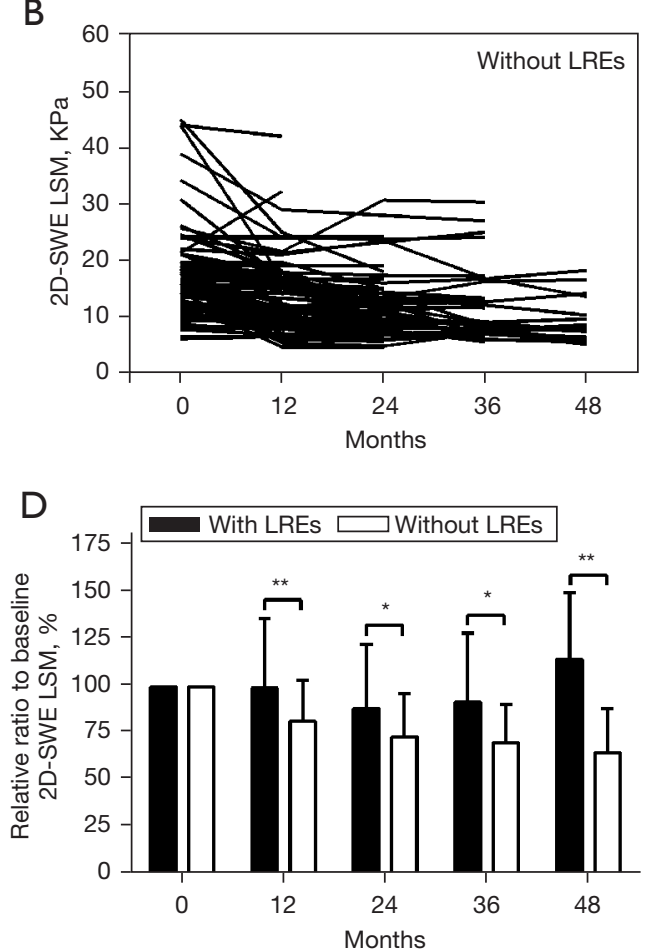

Figure 2 Dynamic changes of LSMs by 2D-SWE in decompensated CHB patients when receiving antiviral therapy. (A-C) The changes of LSMs by 2D-SWE in every participant with LREs (A) or without LREs (B) and their corresponding groups (C). (D) The percentage changes of LSMs by 2D-SWE in the groups that was with LREs and without LREs, respectively. ${ }^{*}, \mathrm{P}<0.05 ;{ }^{* *}, \mathrm{P}<0.01 ;{ }^{* * *}, \mathrm{P}<0.001$. Abbreviations: CHB, chronic hepatitis B; 2D-SWE, two-dimensional shear wave elastography; LRE, liver-related event; LSM, liver stiffness measurement; ns, non-significant.

were $17.0,13.5,11.4,11.3$, and $9.2 \mathrm{kPa}$ at months 0 , $12,24,36$, and 48 , respectively, while the patients who developed LREs presented higher mean LSMs at each corresponding follow-up point of 20.2, 18.1, 16.1, 17.7, and 20.9, respectively (Figure 2C). Moreover, compared with pretreated LSMs by 2D-SWE, the largest decrease in LSM by 2D-SWE was observed in patients with LRE at month $24(20.2 \%)$, while in patients without LREs it was observed at month 48 (45.8\%) (Figure 2D). In contrast, for the other follow-up timepoints, the LSMs showed a slightly decreasing trend in the patients without LREs while a fluctuating drift appeared in those with LREs (Figure 2D). And we found that there were statistically significant differences in LSMs between the two groups with and without LREs $(\mathrm{F}=3.36, \mathrm{P}<0.001)$ and across timepoints $(\mathrm{F}=-0.15, \mathrm{P}<0.001)$, with a significant interaction between group and time $(\mathrm{F}=0.081, \mathrm{P}=0.0075)$ using the two-way repeated measures ANOVA analysis.

\section{Predictors of progression to LREs}

The Kaplan-Meier analysis of all patients divided by different levels of parameters of 2D-SWE monitoring is shown in Figure $3 A-3 F$. The patients in the top 50th quartile of pretreated 2D-SWE LSM, $\Delta \%$ 2D-SWE LSM pre-virus control, or $\Delta \%$ 2D-SWE LSM virus controlpost possessed similar prognostic values as the bottom 50th quartile of the same parameters (Figure $3 A-3 C$ ). However, lower $\Delta \%$ previous yearly changes of $2 \mathrm{D}-\mathrm{SWE}$ LSM, and post-treatment 2D-SWE LSM and higher decreases $\Delta \%$ 2D-SWE LSM pre-post indicated better prognosis than higher values of the same parameters $(\log$-rank $\mathrm{P}<0.001$, $0.0014,<0.001$, respectively, Figure $3 D-3 F$ )

To avoid collinearity, serum fibrosis markers were excluded from the prognostic analysis. Collinearity diagnosis before Cox regression calculation further confirmed that the other variance inflation factors were 

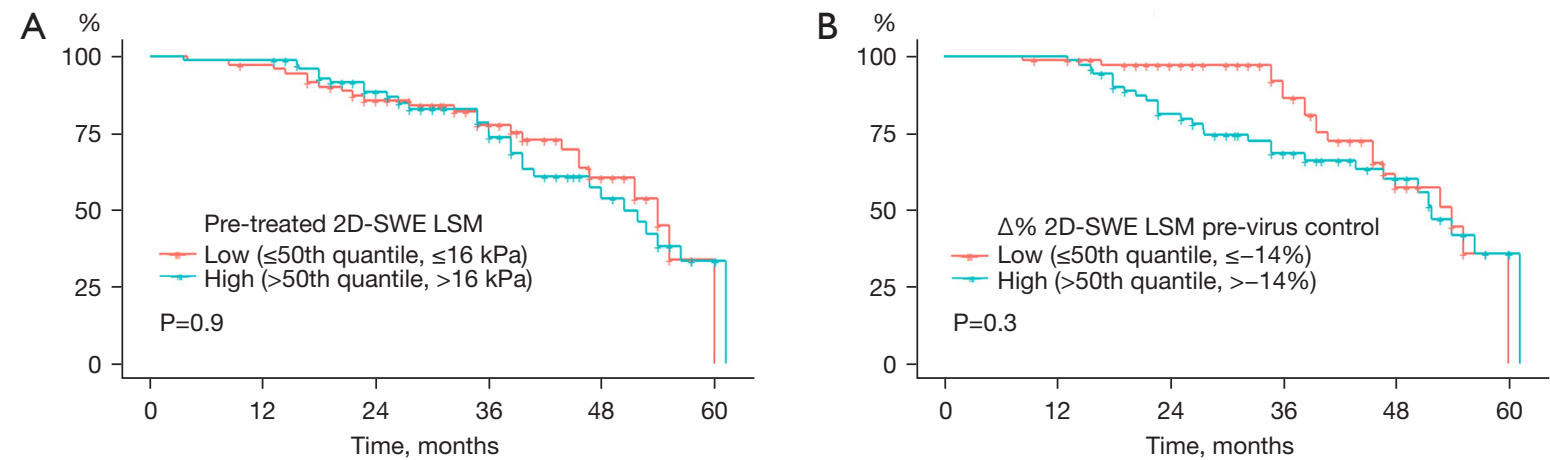

\begin{tabular}{|c|c|c|c|c|c|}
\hline \multicolumn{6}{|c|}{ At risk } \\
\hline Low & 72 & 69 & 55 & 35 & 17 \\
\hline High & 77 & 76 & 55 & 33 & 16 \\
\hline
\end{tabular}

\begin{tabular}{|c|c|c|c|c|c|c|}
\hline \\
\hline $\begin{array}{l}\text { At IISK } \\
\text { Low }\end{array}$ & 70 & 70 & 57 & 34 & 14 & 1 \\
\hline High & 75 & 73 & 52 & 33 & 19 & 5 \\
\hline
\end{tabular}

C $\%$

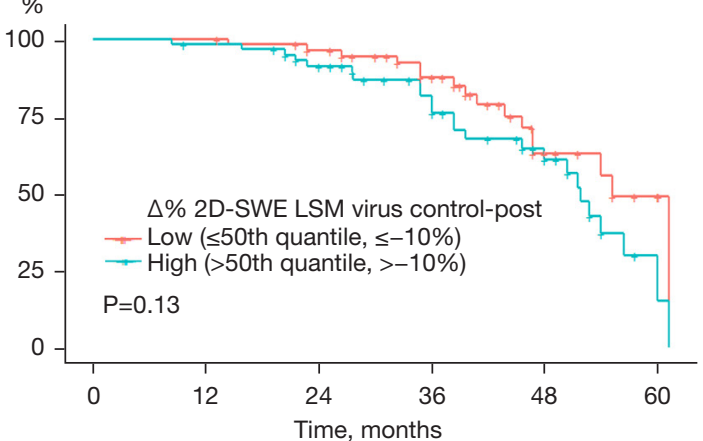

D \%

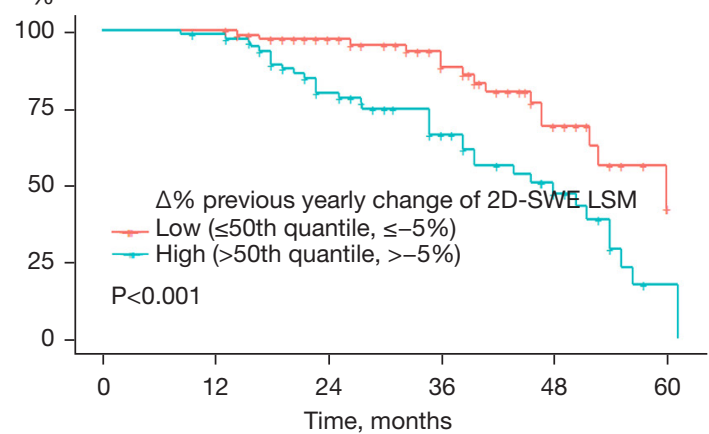

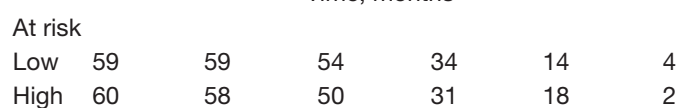

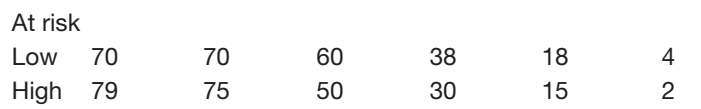

E \%

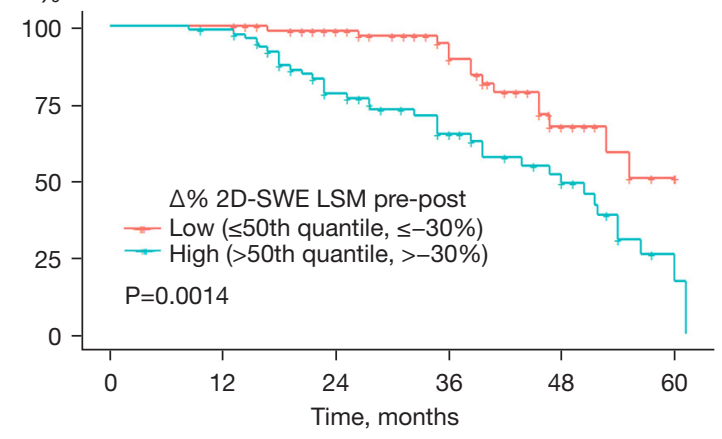

F \%

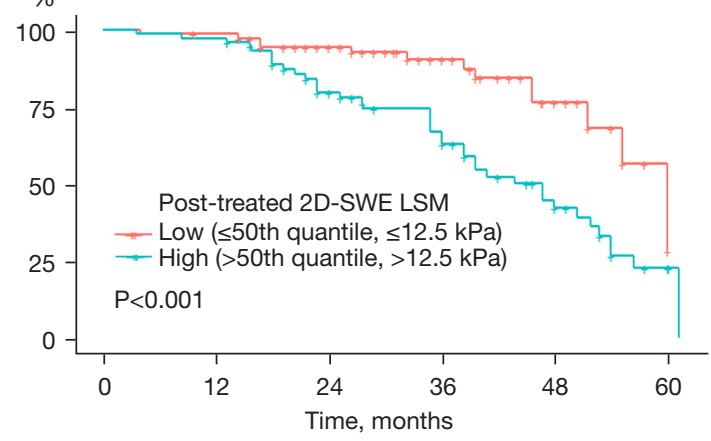

\begin{tabular}{|c|c|c|c|c|c|}
\hline At ris & & & & & \\
\hline Low & 72 & 72 & 62 & 37 & 15 \\
\hline High & 77 & 72 & 48 & 31 & 18 \\
\hline
\end{tabular}

\begin{tabular}{|c|c|c|c|c|}
\hline At risk & & & & \\
\hline Low 74 & 72 & 60 & 33 & 16 \\
\hline High & 73 & 50 & 35 & 17 \\
\hline
\end{tabular}

Figure 3 Kaplan-Meier analysis of developing liver-related events according to parameters derived from 2D-SWE sequential detection. (A) Pre-treated 2D-SWE LSM; (B) $\Delta \%$ 2D-SWE LSM pre-viral control, percent change of LSM at the HBV virus control from baseline; (C) $\Delta \%$ 2D-SWE LSM viral control-post, percent change of LSM at the last follow-up from the time of viral control. The statistical analysis was restricted to those 119 patients who achieved virus control before the end of the follow-up; (D) $\Delta \%$ previous yearly changes of 2D-SWE LSM, percent change of LSM at the last follow-up from the last second. For those who developed liver-related events or dropped out in the first year, their previous yearly changes of 2D-SWE LSM would be considered as $0 \%$ for the statistical analysis; (E) $\Delta \%$ 2D-SWE LSM pre-post, percent change of LSM at the last follow-up from baseline. For those who developed liver-related events or dropped out in the first year, their $\Delta \% 2 \mathrm{D}-\mathrm{SWE}$ LSM pre-post would be considered as $0 \%$ for the statistical analysis; (F) post-treated 2D-SWE LSM. The last 2D-SWE LSM before the last follow-up. If the follow-up period of patients was less than 1 year, the post-treated 2D-SWE LSM would be considered the same as the LSMs by 2D-SWE at the baseline for the statistical analysis. Abbreviations: 2D-SWE, two-dimensional shear wave elastography; LSM, liver stiffness measurement. 
Table 2 Predictors of liver-related events by univariate and multivariate analysis

\begin{tabular}{|c|c|c|c|c|}
\hline Factors & \multicolumn{2}{|c|}{ Univariate analysis } & \multicolumn{2}{|c|}{ Multiple analysis } \\
\hline Male sex & $1.33(0.69-2.56)$ & 0.34 & - & - \\
\hline Age $>55$ years & $1.31(0.75-2.28)$ & 0.39 & - & - \\
\hline Baseline HBsAg levels, $\log _{10} 1 \mathrm{U} / \mathrm{mL}$ & $0.84(0.67-1.05)$ & 0.13 & - & - \\
\hline Baseline HBeAg positive & $1.13(0.63-2.01)$ & 0.67 & - & - \\
\hline Baseline Child-Pugh scores & $1.01(0.81-1.26)$ & 0.91 & - & - \\
\hline Pre-treat 2D-SWE LSM, kPa & $1.03(1.01-1.06)$ & 0.019 & $0.92(0.84-1.00)$ & 0.052 \\
\hline Post-treat 2D-SWE LSM, kPa & $1.05(1.02-1.08)$ & 0.0007 & $1.11(1.04-1.18)$ & 0.0026 \\
\hline$\Delta \%$ previous yearly changes of $2 \mathrm{D}-\mathrm{SWE}$ LSM & $12.33(3.15-48.24)$ & 0.0003 & $5.34(0.34-84.27)$ & 0.230 \\
\hline$\Delta \%$ 2D-SWE LSM virus control-post & $2.21(0.59-8.29)$ & 0.24 & $2.14(0.21-21.84)$ & 0.520 \\
\hline Post-/pre-treated ALT ratio & $1.02(0.98-1.06)$ & 0.38 & - & - \\
\hline Post-/pre-treated total bilirubin ratio & $1.10(1.02-1.19)$ & 0.017 & $1.06(0.69-1.63)$ & 0.800 \\
\hline Post-/pre-treated PLT ratio & $0.56(0.36-0.86)$ & 0.0092 & $0.48(0.20-1.18)$ & 0.100 \\
\hline
\end{tabular}

$\triangle \%$ 2D-SWE LSM pre-post: percent change of LSM at the last follow-up from baseline; $\Delta \% 2 \mathrm{D}-\mathrm{SWE}$ LSM pre-viral control: percent change of LSM at the HBV virus control from baseline; $\triangle \%$ previous yearly changes of 2D-SWE LSM: percent change of LSM at the last follow-up from the last second; $\triangle \%$ 2D-SWE LSM viral control-post: percent change of LSM at the last follow-up from the time of viral control. Abbreviations: ALT, alanine aminotransferase; Cl, confidence interval; 2D-SWE, two-dimensional shear wave elastography; $\mathrm{HBeAg}$, hepatitis B envelope antigen; HBsAg, hepatitis B surface antigen; HBV, hepatitis B virus; HR, hazard ratio; LSM, liver stiffness measurement; PLT, platelet.

$<10$ and that the tolerance was over 0.1. Univariate and multivariate Cox regression analyses were performed to explore the association between 2D-SWE monitoring parameters and the occurrence of LREs. On univariate analysis, pre-treatment 2D-SWE LSM [hazard ratio (HR) $=1.03 ; 95 \%$ confidence interval $(\mathrm{CI}): 1.01-1.06, \mathrm{P}=0.019$ ], post-treatment 2D-SWE LSM (HR $=1.05$; 95\% CI: $1.02-$ $1.08, \mathrm{P}=0.0007), \Delta \% 2 \mathrm{D}-\mathrm{SWE} \mathrm{LSM}$ pre-post $(\mathrm{HR}=2.74$; 95\% CI: 1.29-5.83, $\mathrm{P}=0.0086), \Delta \%$ 2D-SWE LSM previrus control ( $\mathrm{HR}=3.63$; 95\% CI: 1.43-9.18, $\mathrm{P}=0.0065)$, $\Delta \%$ previous yearly changes of $2 \mathrm{D}-\mathrm{SWE}$ LSM $(\mathrm{HR}=12.33$; 95\% CI: $3.15-48.24, \mathrm{P}=0.0003)$, post-/pretreated total bilirubin ratio (HR $=1.10$; $95 \% \mathrm{CI}: 1.02-1.19, \mathrm{P}=0.017)$ and post-/pretreated PLT ratio (HR $=0.56$; 95\% CI: $0.36-0.86$, $\mathrm{P}=0.0092$ ) were significantly associated with the occurrence of LREs (Table 2). Multivariable analysis revealed that posttreatment 2D-SWE LSM (HR $=1.11$; 95\% CI: 1.04-1.18, $\mathrm{P}=0.0026$ ) was the only independent risk factor for the occurrence of LREs (Table 2).

\section{Predictive accuracy of the post-treatment 2D-SWE LSM in comparison with the other parameters}

ROC curve analysis was applied to estimate the clinical value of those 2D-SWE-based predictors and the serum fibrosis markers. The AUC of the post-treatment 2D-SWE LSM was the largest (AUC $=0.75, \mathrm{P}<0.001$ ) among all the 2D-SWE-based variables investigated, followed by $\Delta \%$ previous yearly changes in 2D-SWE LSM (AUC $=0.69$, $\mathrm{P}=0.0015$ ), and $\Delta \% 2 \mathrm{D}-\mathrm{SWE}$ LSM pre-post (AUC $=0.68$, $\mathrm{P}=0.001$ ) (Figure $4 A$ ). For comparison with serum fibrosis markers, post-treatment 2D-SWE LSM had the highest accuracy with an AUC of 0.75 , which was significantly superior to the AUCs of FN (AUC $=0.494, \mathrm{P}=0.79$ ), HA (AUC $=0.429, \mathrm{P}=0.091$ ), PIIINP (AUC =0.609, $\mathrm{P}=0.0258$ ), $\mathrm{LN}(\mathrm{AUC}=0.609, \mathrm{P}=0.007), \mathrm{CIV}(\mathrm{AUC}=0.565, \mathrm{P}=0.017)$ 
A

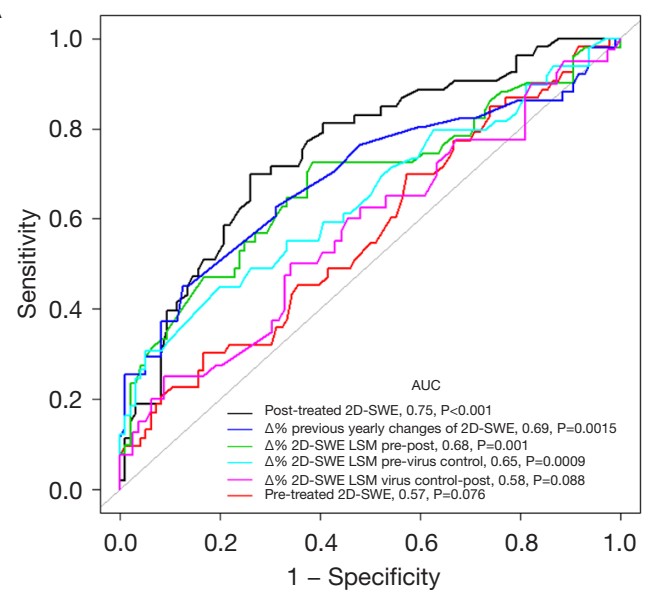

B

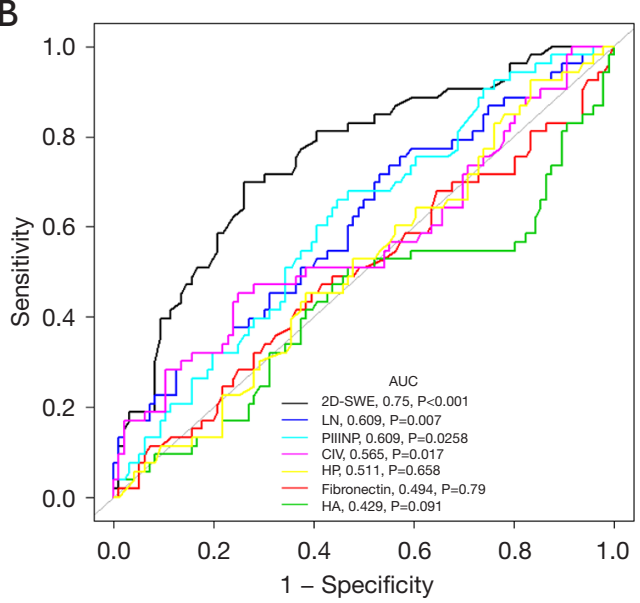

Figure 4 Comparisons of predicting value of between parameters derived from 2D-SWE (A) and serum fibrosis markers (B). Abbreviations: AUC, area under the receiver operating characteristic curve; CIV, collagen type IV; 2D-SWE, two-dimensional shear wave elastography; HA, hyaluronic acid; HP, haptoglobin; LN, laminin; LSM, liver stiffness measurement; PIIINP, procollagen III amino terminal propeptide.

and $\mathrm{HP}(\mathrm{AUC}=0.511, \mathrm{P}=0.658)$ (Figure $4 B$ ). When applying post-treatment $2 \mathrm{D}$-SWE LSM to predict the nonoccurrence of LREs, post-treatment 2D-SWE LSM had the highest AUC to predict the nonoccurrence of LREs for a cutoff value $<14.3 \mathrm{kPa}$, with $74 \%$ specificity and $70 \%$ sensitivity.

\section{Discussion}

Several studies have demonstrated the key role of 2D-SWE in hepatic fibrosis severity, monitoring disease progression and predicting outcomes. However, in contrast to most existing data demonstrating the predictive role of the baseline 2D-SWE LSM levels in advanced liver disease $(15,16)$, our study was the first to investigate the prognostic value of the 2D-SWE LSM level as well as its dynamic changes in patients with CHB-related decompensated cirrhosis undergoing antiviral therapy.

Our data showed that among patients with the improved prognosis of CHB-related decompensated cirrhosis, 2D-SWE LSM decreased substantially from the pre-treatment baseline to the follow-up after NUC initiation, while for those with poor outcomes, 2D-SWE LSM represented a fluctuating trend. This is similar to a previous report that aimed to estimate on-treatment changes in liver stiffness utilizing another elastography as transient elastography in 438 patients with compensated cirrhosis undergoing antiviral therapy (5). In both this report and another study with paired biopsy on antiviral therapy for LSM changes in CHB patients with various fibrosis severities (18), a biphasic pattern of LSM decline of transient elastography in CHB patients was raised as a rapidly improving phase during the first 6 months of treatment and a slowly improving phase thereafter until 1824 months. Interestingly, the decreasing pattern of 2D-SWE LSM in our study for decompensated cirrhosis stage also presented as a sluggish slow-down and mostly existed in patients with improved outcomes. As CHB progressed to the decompensated phase, the fibrosis of which was unlikely to reverse, the LSM decline on antiviral treatment may be attributed to several reasons. First, fibrosis assessments of 2D-SWE would be affected by liver inflammation activities; for the fibrosis stage ahead of cirrhosis, increased liver inflammation leads to overstaging of fibrosis (9); therefore, it may also contribute to higher LSMs in the decompensated stage. Inflammation regressed as potent inhibition of $\mathrm{HBV}$ DNA replication by antiviral agents, causing a decrease in LSM of 2D-SWE. Second, intrahepatic cholestasis such as jaundice and elevated bilirubin, which are common in liver decompensated cirrhosis has been identified as another factor influencing LSMs by 2D-SWE. For patients with the same $\mathrm{F} 4$ stage according to the Metavir fibrosis scoring system, CHB patients with abnormal bilirubin levels had higher LSMs and elevated bilirubin levels had a positive correlation with LSMs (correlation coefficient of 0.435) (19). Antiviral therapy may also decrease LSMs by improving bilirubin levels. Third, portal hypertension is one of the most important pathophysiological mechanisms in LRE 
development, and LSM by 2D-SWE is highly correlated with hepatic venous pressure gradient values in cirrhotic patients with ascites (20). There has been mounting evidence demonstrating that long-term oral entecavir antiviral therapy reduces cirrhotic events, including the regression of esophageal varices $(21,22)$. Taken together, antiviral therapy significantly reduces disease progression in decompensated cirrhosis and improves inflammation, intrahepatic cholestasis and portal hypertension, which may be reflected in the LSM of 2D-SWE.

Remarkably, the post-treatment LSM of 2D-SWE, but not the pre-treatment level or dynamic changes in LSM, appeared to be a superior predictor of LRE in our study. In patients without decompensated cirrhosis, the baseline levels of 2D-SWE or quick liver stiffness response seemed to be useful markers for predicting LREs or remission of disease $(8,18)$. This may be because pre-treatment 2D-SWE simply reflects the baseline status, whereas the post-treatment LSM level may represent the combination of baseline and the treatment response, and the treatment response has been reported to have high heterogeneity $(23,24)$. Because the risks of LREs remained high in patients with cirrhosis during entecavir therapy (22), sequential and regular monitoring of $2 \mathrm{D}-\mathrm{SWE}$ is useful for identifying the risk of LRE development in these on-treatment patients, even in the decompensated stage.

The present study has the following limitations. First, for decompensated cirrhosis, we were unable to evaluate histologic fibrosis severity or its changes after treatment, which may be helpful in revealing the mechanism underlying the changes in LSM after treatment. Second, not all patients completed a sufficiently long-term follow-up. Third, further verification in a multicenter study with a larger sample size is warranted. Fourth, the LSM measurements when LRE occurred was important results to support our conclusion. However, when the LREs such as esophageal varices bleeding, spontaneous bacterial peritonitis, hepatic encephalopathy, hepatopulmonary syndrome, hepatorenal syndrome and liver failure occurred, the patients were advised to receive emergency rescue nearby instead of returning to our center for 2D-SWE measurements due to the ethnicity. Those who underwent LRE dropped out of the SWE protocol because LRE included gastric or esophageal varices bleeding, spontaneous bacterial peritonitis, hepatic encephalopathy, hepatopulmonary syndrome, hepatorenal syndrome and liver failure, hepatocellular carcinoma, or liver-related death, and some of those who underwent LRE received liver transplantation, transjugular intrahepatic portosystemic shunt (Tips) therapy, liver tumor resection or radiofrequency ablation, which would cause significant change in LSM that might not be associated with SWE monitoring for predicting LRE occurrence. Due to the complicated situation for these patients, the current SWE monitoring protocol should be further adjusted and estimated. Finally, the current study demonstrated 2D-SWE as a more accurate noninvasive tool than serum fibrosis markers for monitoring patients with decompensated cirrhosis and CHB during antiviral treatment. It may facilitate identification of patients at high risks after initiating antiviral treatment for a more intensive intervention and those at low risk for regular management. Nevertheless, how LSM of 2D-SWE monitoring can help to adjust treatment regimens remains unknown.

In conclusion, we assessed changes in 2D-SWE LSM and their predictive role in the poor prognosis of patients with CHB-related decompensated cirrhosis during antiviral treatment. Our data provides novel evidence that posttreatment LSM of 2D-SWE is useful for predicting future LRE development in such patients and that this parameter is a more accurate approach than baseline and dynamic changes in LSM of 2D-SWE, levels of liver functions or serum fibrosis markers at times before decompensated event reoccurrence. According to our study, routine measurements of $2 \mathrm{D}-\mathrm{SWE}$ may provide valuable complementary information to other conventional indexes for predicting disease prognosis in these patients.

\section{Acknowledgments}

Funding: This study was supported by Postdoctoral Research Foundation of China (2020M683128) and the National Natural Science Foundation of China (No. 81870404, 81670518 and No. 81170392).

\section{Footnote}

Reporting Checklist: The authors have completed the STARD reporting checklist. Available at https://dx.doi. org/10.21037/atm-21-3142

Data Sharing Statement: Available at https://dx.doi. org/10.21037/atm-21-3142

Peer Review File: Available at https://dx.doi.org/10.21037/ atm-21-3142 
Conflicts of Interest: All authors have completed the ICMJE uniform disclosure form (available at https://dx.doi. org/10.21037/atm-21-3142). The authors have no conflicts of interest to declare.

Etbical Statement: The authors are accountable for all aspects of the work in ensuring that questions related to the accuracy or integrity of any part of the work are appropriately investigated and resolved. The study was conducted in accordance with the Declaration of Helsinki (as revised in 2013). The study protocol was approved by the institutional ethics committee of The First Affiliated Hospital, Sun Yat-sen university $\{[2014] 112\}$. All participants signed informed consent before taking part and agreed on their relevant data in this project to be analyzed.

Open Access Statement: This is an Open Access article distributed in accordance with the Creative Commons Attribution-NonCommercial-NoDerivs 4.0 International License (CC BY-NC-ND 4.0), which permits the noncommercial replication and distribution of the article with the strict proviso that no changes or edits are made and the original work is properly cited (including links to both the formal publication through the relevant DOI and the license). See: https://creativecommons.org/licenses/by-nc-nd/4.0/.

\section{References}

1. Lee HM, Banini BA. Updates on Chronic HBV: Current Challenges and Future Goals. Curr Treat Options Gastroenterol 2019;17:271-91.

2. D'Amico G, Garcia-Tsao G, Pagliaro L. Natural history and prognostic indicators of survival in cirrhosis: a systematic review of 118 studies. J Hepatol 2006;44:217-31.

3. de Franchis R; Baveno VI Faculty. Expanding consensus in portal hypertension: Report of the Baveno VI Consensus Workshop: Stratifying risk and individualizing care for portal hypertension. J Hepatol 2015;63:743-52.

4. Wang JH, Chuah SK, Lu SN, et al. Baseline and serial liver stiffness measurement in prediction of portal hypertension progression for patients with compensated cirrhosis. Liver Int 2014;34:1340-8.

5. Wu S, Kong Y, Piao H, et al. On-treatment changes of liver stiffness at week 26 could predict 2-year clinical outcomes in HBV-related compensated cirrhosis. Liver Int 2018;38:1045-54.

6. Thiele M, Hugger MB, Kim Y, et al. 2D shear wave liver elastography by Aixplorer to detect portal hypertension in cirrhosis: An individual patient data meta-analysis. Liver Int 2020;40:1435-46.

7. Herrmann E, de Lédinghen V, Cassinotto C, et al. Assessment of biopsy-proven liver fibrosis by twodimensional shear wave elastography: An individual patient data-based meta-analysis. Hepatology 2018;67:260-72.

8. Wu M, Wu L, Jin J, et al. Liver Stiffness Measured with Two-dimensional Shear-Wave Elastography Is Predictive of Liver-related Events in Patients with Chronic Liver Disease Due to Hepatis B Viral Infection. Radiology 2020;295:353-60.

9. Ye J, Wang W, Feng S, et al. Precise fibrosis staging with shear wave elastography in chronic hepatitis B depends on liver inflammation and steatosis. Hepatol Int 2020;14:190-201.

10. Shiha G, Ibrahim A, Helmy A, et al. Asian-Pacific Association for the Study of the Liver (APASL) consensus guidelines on invasive and non-invasive assessment of hepatic fibrosis: a 2016 update. Hepatol Int 2017;11:1-30.

11. Kamath PS, Kim WR; Advanced Liver Disease Study Group. The model for end-stage liver disease (MELD). Hepatology 2007;45:797-805.

12. Marrero JA, Kudo M, Venook AP, et al. Observational registry of sorafenib use in clinical practice across ChildPugh subgroups: The GIDEON study. J Hepatol 2016;65:1140-7.

13. Hou J, Wang G, Wang F, et al. Guideline of Prevention and Treatment for Chronic Hepatitis B (2015 Update). J Clin Transl Hepatol 2017;5:297-318.

14. Chinese Society of Hepatology, Chinese Medical Association; Xu X, Duan Z, et al. Chinese guidelines on the management of ascites and its related complications in cirrhosis. Hepatol Int 2019;13:1-21.

15. European Association for the Study of the Liver. Electronic address: easloffice@easloffice.eu; European Association for the Study of the Liver. EASL Clinical Practice Guidelines for the management of patients with decompensated cirrhosis. J Hepatol 2018;69:406-60.

16. Thiele M, Hugger MB, Kim Y, et al. 2D shear wave liver elastography by Aixplorer to detect portal hypertension in cirrhosis: An individual patient data meta-analysis. Liver Int 2020;40:1435-46.

17. Gad MAM, Eraky TE, Omar HM, et al. Role of real-time shear-wave elastogarphy in differentiating hepatocellular carcinoma from other hepatic focal lesions. Eur J Gastroenterol Hepatol 2021;33:407-14.

18. Kong Y, Sun Y, Zhou J, et al. Early steep decline of liver stiffness predicts histological reversal of fibrosis in chronic 
hepatitis B patients treated with entecavir. J Viral Hepat 2019;26:576-85.

19. Guo H, Liao M, Jin J, et al. How intrahepatic cholestasis affects liver stiffness in patients with chronic hepatitis B: a study of 1197 patients with liver biopsy. Eur Radiol 2020;30:1096-104.

20. Kim TY, Jeong WK, Sohn JH, et al. Evaluation of portal hypertension by real-time shear wave elastography in cirrhotic patients. Liver Int 2015;35:2416-24.

21. Jwa HY, Cho YK, Choi EK, et al. Regression of esophageal varices during entecavir treatment in patients with hepatitis-B-virus-related liver cirrhosis. Clin Mol Hepatol 2016;22:183-7.

22. Su TH, Hu TH, Chen CY, et al. Four-year entecavir

Cite this article as: Ye J, Huang Y, Sun Y, Shao C, Zhang S, Wang W, Zhong B. Dynamic monitoring with shear wave elastography predicts outcomes of chronic hepatitis B patients with decompensated cirrhosis. Ann Transl Med 2021;9(21):1613. doi: 10.21037/atm-21-3142 therapy reduces hepatocellular carcinoma, cirrhotic events and mortality in chronic hepatitis B patients. Liver Int 2016;36:1755-64.

23. Arends P, Sonneveld MJ, Zoutendijk R, et al. Entecavir treatment does not eliminate the risk of hepatocellular carcinoma in chronic hepatitis B: limited role for risk scores in Caucasians. Gut 2015;64:1289-95.

24. Papatheodoridis GV, Manolakopoulos S, Touloumi $\mathrm{G}$, et al. Virological suppression does not prevent the development of hepatocellular carcinoma in HBeAgnegative chronic hepatitis $B$ patients with cirrhosis receiving oral antiviral(s) starting with lamivudine monotherapy: results of the nationwide HEPNET. Greece cohort study. Gut 2011;60:1109-16. 
Appendix 1: Questionnaire of chronic hepatitis B disease for the clinical trial registered in the Chinese Clinical Trial Register [ChiCTR-DCD-15006000]

\section{Identification Number:}

Time of enrollment (DD/MM/YY):

Part 1. Socio-demographic characteristics of the participants:

1.1 Name: ; Age: in years.

1.2 Gender: male/female

1.3 Date of birth (DD/MM/YY):

1.4 Occupation: Employed, specify

1.5 Address: City

1.6 Phone number 1 : ; Unemployed

; Sub-city ; Woreda

1.7 Education status: illiteracy [1]/primary school [2]/middle school [3]/junior college [4]/university and above [5]

1.8 Family's monthly income:

1.9 Number of family members:

Part 2. Diagnosis at the first interview:

2.1 HBV DNA positive: Yes/No. If yes, when was it diagnosed? weeks. The name of health center at diagnosis HBV DNA level

2.2 HBsAg positive: Yes/No. If yes, when was it diagnosed? , HBsAg level

2.3 Hepatic decompensation. Yes/No, gastric or esophageal varices bleeding, spontaneous bacterial peritonitis, hepatic encephalopathy, hepatopulmonary syndrome, hepatorenal syndrome, liver failure. If yes, when was it diagnosed? weeks. The name of health center at diagnosis

2.4 Hepatocellular carcinoma Yes/No. If yes, when was it diagnosed? weeks. The name of health center at diagnosis

\section{Part 3. Medical history:}

3.1 History of present illness:

If yes, when was it diagnosed?

3.2 Previous medical history:

If yes, when was it diagnosed? weeks. The name of health center at diagnosis

3.3 The drug was taking weeks. The name of health center at diagnosis

3.4 The history of surgery and blood transfusion:

3.5 The history of drinking: never/occasionally/frequently, drinking for years, g alcohol/week, abstinence for years

3.6 The history of smoking: never/have ( $<10$ cigarettes a day/10-20 cigarettes a day/>20 cigarettes a day), smoking for years, quitting for smoking for years

3.7 Family history:

Hepatitis B: Yes/No, father/mother/brothers and sisters/children

Fatty liver: Yes/No, father/mother/brothers and sisters/children

Obesity: Yes/No, father/mother/brothers and sisters/children

Hypertension: Yes/No, father/mother/brothers and sisters/children

Diabetes mellitus: Yes/No, father/mother/brothers and sisters/children

Coronary heart disease: Yes/No, father/mother/brothers and sisters/children

Others: 
Part 4. Anthropometric indices:

4.1 Blood pressure mmHg (left/right arm) Time

4.2 Height $\mathrm{cm}$; Weight $\mathrm{kg}$; BMI (body mass index $)=$ weight $(\mathrm{kg}) /$ height $(\mathrm{m} 2)=$

4.3 Waist circumference $\mathrm{cm}$; Abdomen circumference cm; Hip circumference cm; Waist-hip ratio

4.4 Grip $\mathrm{kg}$
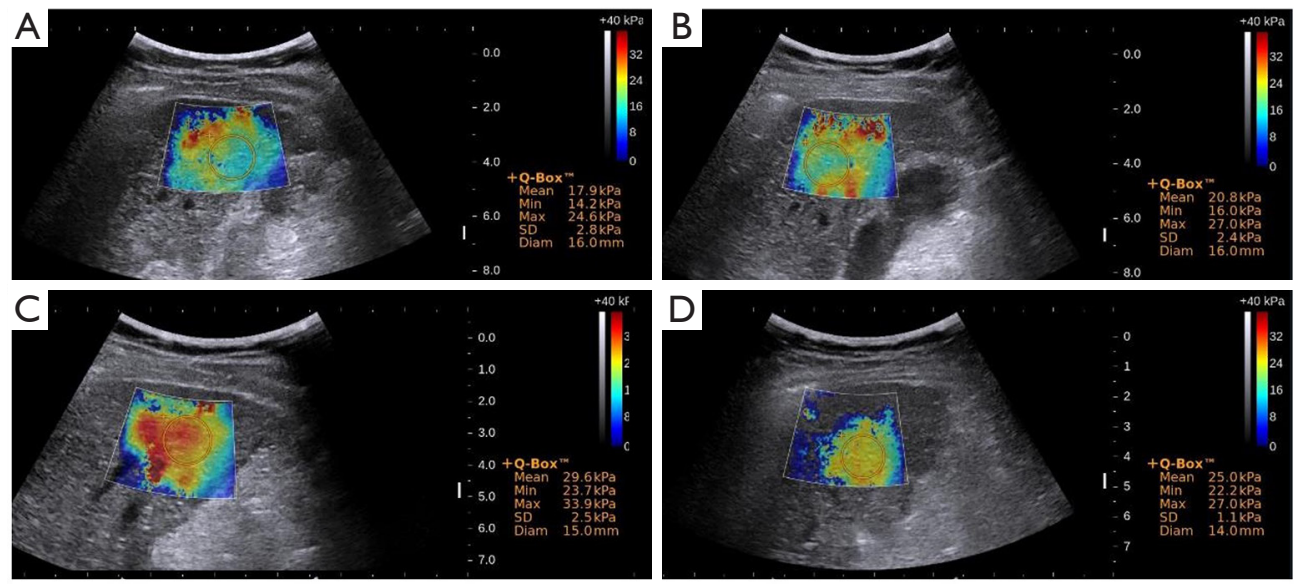

Figure S1 Representative images of two-dimensional shear-wave elastography (2D-SWE) exhibit liver stiffness measurements in a 65 -year-old female with decompensated cirrhosis caused by chronic hepatitis B. Rectangular elasticity box $(4 \mathrm{~cm} \times 3 \mathrm{~cm})$ was placed 1-2 $\mathrm{cm}$ under liver capsule in parenchyma area free of large vessels. Circular region of interest was $2 \mathrm{~cm}$ in diameter and was positioned in center of 2D-SWE elasticity box possibly. The operators aimed to achieve homogeneous color filling of the SWE ROI placed on the most homogeneous, stable elastogram area. (A) Before antiviral treatments; (B) increased liver stiffness after antiviral treatment for 3 years. Representative images of 2D-SWE exhibit liver stiffness measurements in another 58-year-old female; (C) before antiviral treatments; (D) decreased liver stiffness after antiviral treatment for 1 year.

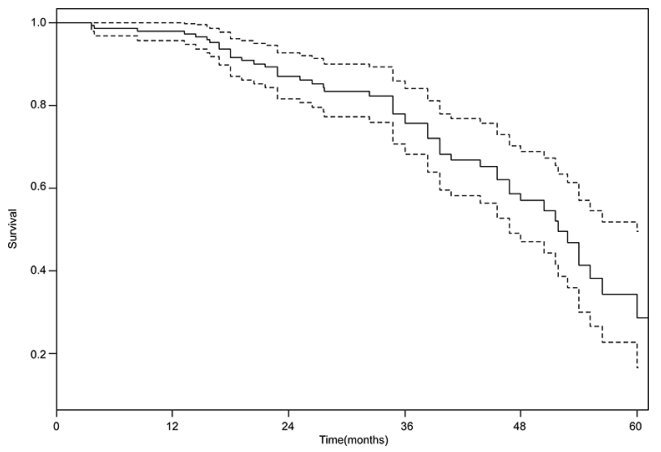

Figure S2 The cumulative rates of remaining free from the occurrence liver related events in Chronic hepatitis B related decompensated cirrhosis. The two dashed lines represent the $95 \%$ confident interval of the survival curves. 
Table S1 Characteristics of patients with HBV-related decompensated cirrhosis at the last measurement of 2D-SWE and serum fibrosis markers

\begin{tabular}{|c|c|c|c|}
\hline Characteristic & $\begin{array}{l}\text { Without liver-related events developed } \\
\text { after follow-up }(\mathrm{N}=96)\end{array}$ & $\begin{array}{l}\text { With liver-related events developed after } \\
\text { follow-up }(\mathrm{N}=53)\end{array}$ & $P$ value \\
\hline HBV DNA >20 IU/mL, \% & 0 & 1.9 & 0.600 \\
\hline $\mathrm{HBsAg}, \log _{10} \mathrm{IU} / \mathrm{mL}$ & $2.6 \pm 1.1$ & $2.4 \pm 1.2$ & 0.320 \\
\hline Time to HBV virus control, months & $6.8 \pm 4.2$ & $7.2 \pm 5.5$ & 0.220 \\
\hline ALT, IU/L & $26.0(19.0-34.0)$ & $29.0(22.0-36.0)$ & 0.097 \\
\hline AST, IU/L & $26.5(22.0-35.2)$ & $35.0(25.8-52.0)$ & 0.088 \\
\hline GGT, IU/L & $28.5(22.0-43.5)$ & $45.0(27.0-70.0)$ & 0.010 \\
\hline Total bilirubin, $\mu \mathrm{mol} / \mathrm{L}$ & $16.7(11.2-21.7)$ & $22.1(12.1-33.9)$ & 0.052 \\
\hline Creatinine, $\mu \mathrm{mol} / \mathrm{L}$ & $81.5(60.4-103.6)$ & $110.8(64.3-138.4)$ & 0.088 \\
\hline Platelets, $10^{3} / \mu \mathrm{L}$ & $155.0 \pm 83.1$ & $130.9 \pm 77.1$ & 0.670 \\
\hline Prothrombin activity, \% & $93.2 \pm 16.7$ & $78.1 \pm 22.6$ & 0.053 \\
\hline \multicolumn{4}{|l|}{ Serum fibrosis markers } \\
\hline Fibronectin, mg/L & $178.6 \pm 31.9$ & $180.1 \pm 33.0$ & 0.820 \\
\hline $\mathrm{HA}, \mathrm{ng} / \mathrm{mL}$ & $91.8(33.0-174.3)$ & $103.8(37.5-169.7)$ & 0.110 \\
\hline
\end{tabular}

Normally and non-normally distributed variables were expressed as mean \pm standard deviation or median (25-75\% quantiles), respectively. 2D-SWE, two-dimensional shear wave elastography; AFP, alpha-fetoprotein; ALP, alkaline phosphatase; ALT, alanine aminotransferase; AST, aspartate transaminase; BMI, body mass index; CIV, collagen type IV; GGT, glutamyl transferase; HA, hyaluronic acid; HBeAg, hepatitis B envelope antigen; HBsAg, hepatitis B surface antigen; HBV, hepatitis B virus; HP, haptoglobin; LN, laminin; LRE, liver-related event; PIIINP, procollagen III amino terminal propeptide. 